\title{
Le monde ouvrier, la photographie
}

Henri Eckert

\section{OpenEdition}

\section{Journals}

Édition électronique

URL : https://journals.openedition.org/itti/1163

DOI : 10.4000/itti.1163

\section{Éditeur}

Université de Poitiers

\section{Référence électronique}

Henri Eckert, « Le monde ouvrier, la photographie », Images du travail, travail des images [En ligne], 2 |

2016, mis en ligne le 01 septembre 2016, consulté le 24 juillet 2021. URL : http://

journals.openedition.org/itti/1163; DOI : https://doi.org/10.4000/itti.1163

Ce document a été généré automatiquement le 24 juillet 2021.

Images du travail, travail des images 


\section{Le monde ouvrier, la photographie}

\section{Henri Eckert}

1 La photographie n'a jamais eu le monopole de la représentation du monde ouvrier. Peintres, dessinateurs, caricaturistes ou propagandistes, entre autres, ont largement contribué à en produire des images, dès avant l'invention de la photographie et par la suite. Mais sans doute faut-il considérer que la photographie a pu se prévaloir de deux avantages décisifs dans la concurrence qui a pu l'opposer aux autres modes de représentation : sa capacité à enregistrer le réel qui se présente devant son objectif et donc à le documenter avec une exactitude plus grande que le peintre, par exemple, et la possibilité dont elle s'est dotée très rapidement de reproduire ses images à l'infini, sur les supports les plus divers, pages de livres ou de revues notamment, ou encore cartes postales. Une photographie porte toujours la trace de quelque chose qui a été - cf. le "ça a été » de Roland Barthes (1980) -, elle peut, de ce fait, porter témoignage de la portion de réel qu'elle restitue et son témoignage a pu très tôt être diffusé largement, dans un souci d'intervention ou, plus modestement, d'alerte, selon les intentions du photographe. 
Illustration 1.

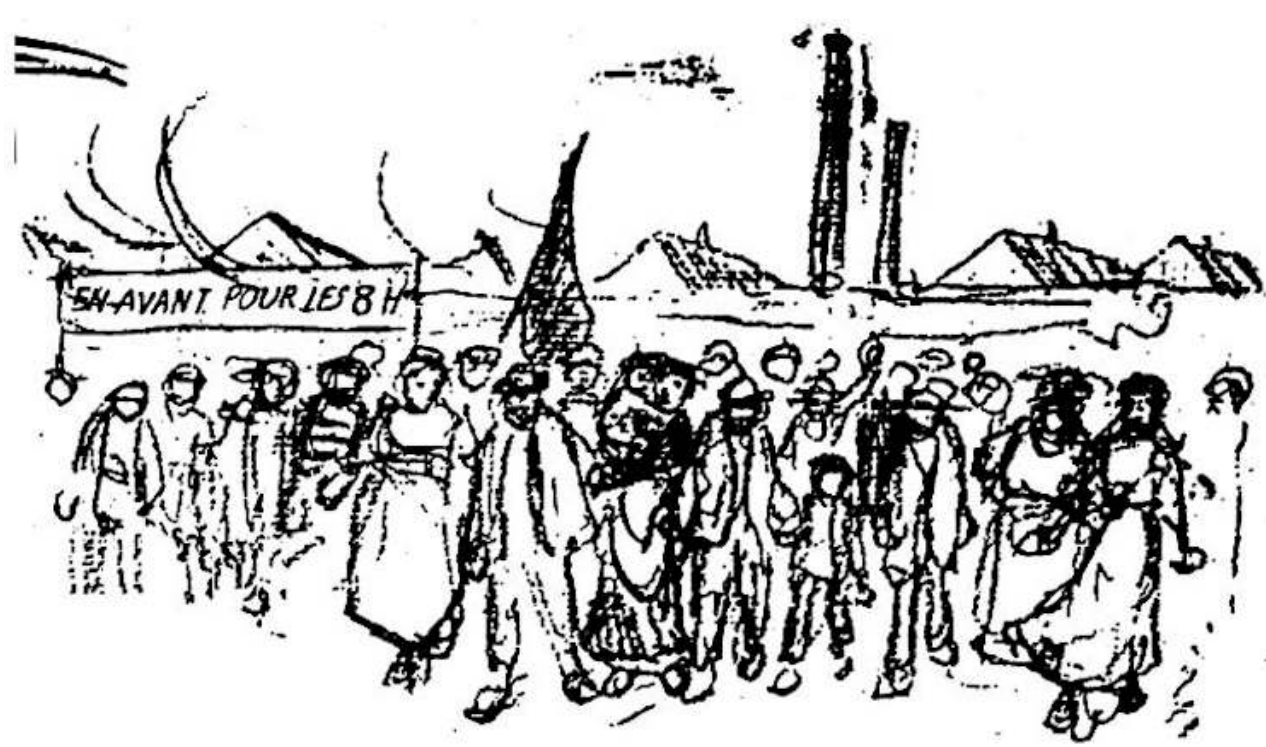

Cf. Pigenet M. et Robert J-L. (2000), «Travailleurs, syndiqués et syndicats dans les dessins de La Voix du Peuple (1900-1914)", Sociétés \& Représentations, n 10, p. 309-322.

2 Si la photographie s'est ainsi proposée d'emblée comme moyen d'agir sur le monde (Benjamin, 1935/2002), elle est aussi apparue à un moment historique qui invitait à rendre compte des profondes évolutions en cours. Le développement industriel est largement amorcé lorsque L. Daguerre ou W.H. Fox Talbot réalisent leurs premières images. Mais il ne s'agit pas là d'une simple concomitance. L'invention de la photographie ne cristallise pas seulement les progrès de la chimie moderne et ne substitue pas seulement un dispositif technique, à la fois chimique et mécanique, à la main du peintre; il y a aussi que son développement s'est calé sur la production en série de marchandises standardisées, caractéristique de la transformation alors en cours du mode de production capitaliste (Rouillé, 2005). Les conditions dans lesquelles celle-ci se réalisait ont très vite attiré l'attention des observateurs les plus divers, des philanthropes aux théoriciens du mouvement social en passant par les médecins ou les sociologues. Si l'enquête utilisait encore avant tout les procédés de l'observation ou du questionnaire, les photographes n'ont pas tardé à fournir des images, quelquefois prises dans les lieux mêmes de production, plus fréquemment à leur périphérie. 
Photographie 1.

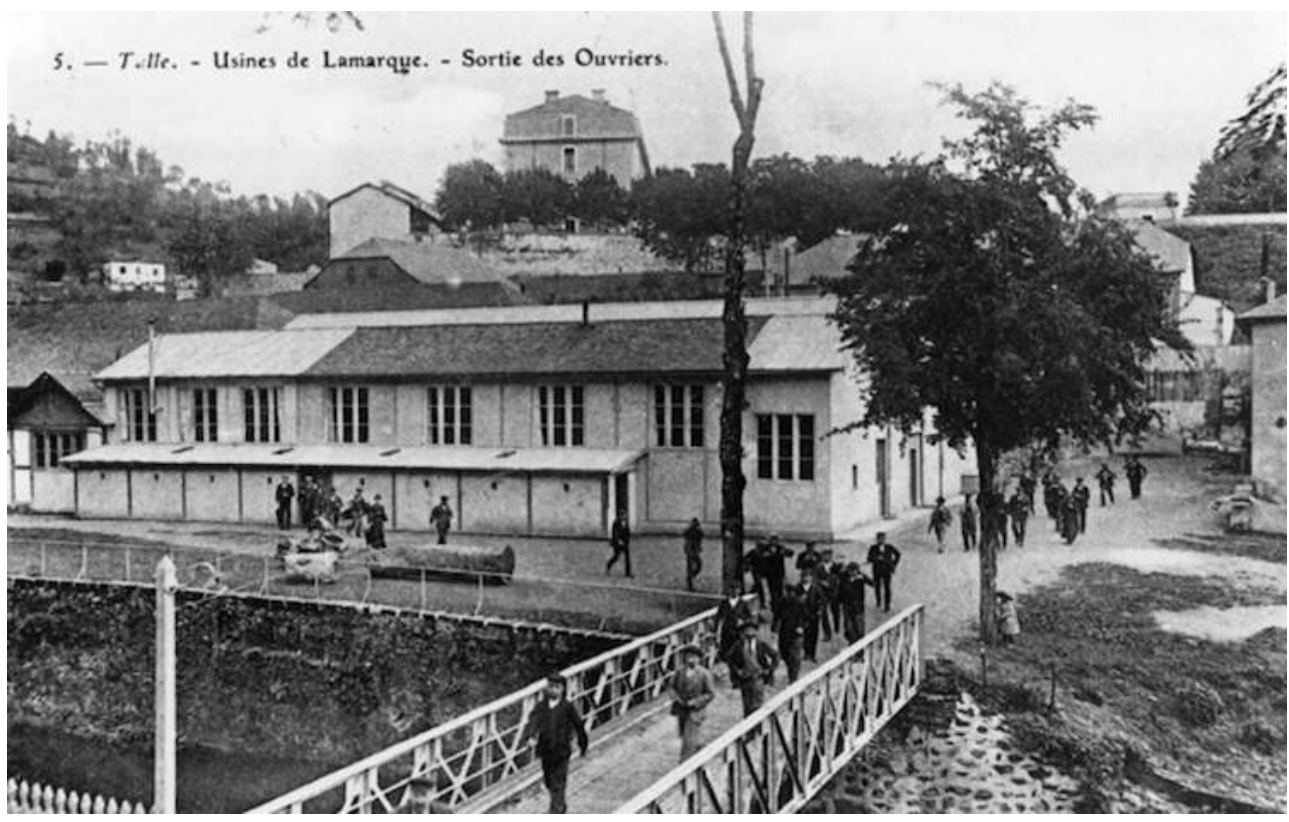

Madelmont M-M., Quincy G. et Touleyrou M. (1981), Le monde ouvrier en Corrèze au XIXe siècle, Tulle, 73

Tout comme le cinéma le fera un peu plus tard - lorsqu'il montrera pour la première fois des ouvriers, des ouvrières en l'occurrence, en filmant La sortie de l'usine Lumière à Lyon (1895) -, la photographie semble avoir volontiers dirigé son objectif sur les sorties d'usine. Les photographes ont certes été sollicités par les propriétaires/patrons d'usines qui leur ont commandé des photos des lieux de production, leur demandant de montrer les installations et les machines, les ouvriers parfois debout à côté d'elles. Les patrons leur ont commandé aussi des photographies sur lesquelles était rassemblé l'ensemble du personnel de l'usine, un peu sur le modèle de la photo de famille... Faut-il voir dans ces pratiques un effet de la réticence à montrer le travail lui-même, les ouvriers/ouvrières, voire les enfants, au travail? Dans les situations concrètes de travail ? Afin d'occulter ou de masquer la dureté des conditions de travail, qui auraient sans doute été visibles sur les photos? L'entretien que nous avons réalisé avec Gérald Bloncourt donne une idée des difficultés qu'a pu rencontrer un photographe qui cherchait à rentrer dans les usines pour photographier les ouvriers sur leur lieu de travail. Mais Gérald Bloncourt nous y parle aussi des ouvriers dans leur cadre de vie ou les luttes! 


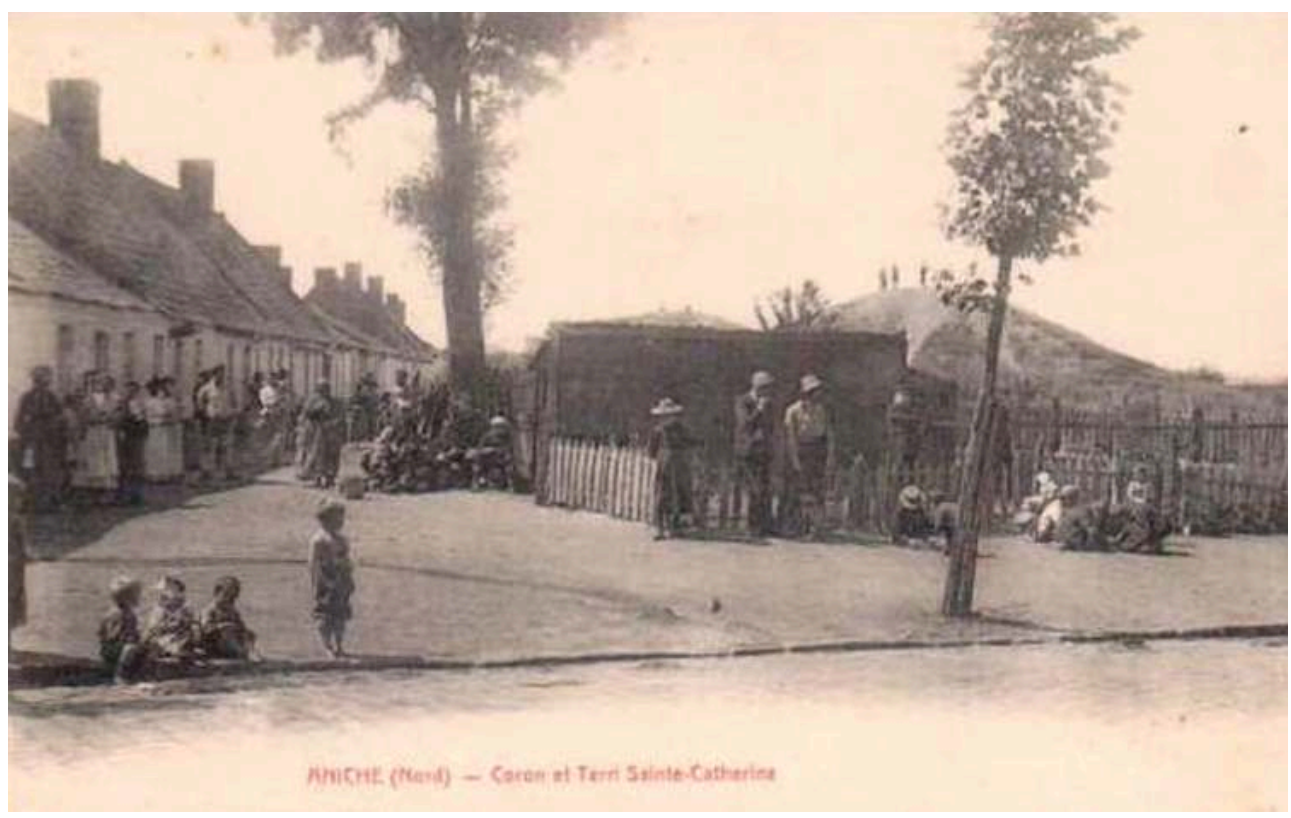

Ces questions, sommairement pointées, dessinent le fond des articles réunis dans notre dossier. Ceux-ci renvoient essentiellement à la période récente - de 1945 à nos jours mais s'organisent autour de cette question plus actuelle que jamais: comment photographier le monde ouvrier? Moins nombreux aujourd'hui, désormais dépassés par les employés, les ouvriers seraient devenus silencieux (Azémar, 1992) ; ne seraientils pas aussi, d'une certaine manière, devenus invisibles? Le monde ouvrier se rétracte, les fermetures d'usines, parfois liées aux difficultés conjoncturelles, plus souvent aux stratégies des firmes à la recherche d'une rentabilité maximale du capital investi, ont entrainé la délocalisation massive d'industries et, en réaction, des grèves, souvent accompagnées de l'occupation des sites de production menacés. Une autre figure ouvrière a fait irruption: celle d'ouvriers menacés de licenciement, surgissant sur le devant de la scène sociale après en avoir été écartés depuis que le discours idéologique majoritaire nous a assurés de l'avènement d'une société postindustrielle... Ils sont là pourtant, à braver les intempéries de la conjoncture, ne serait-ce que parce que certains photographes s'attachent encore à les rencontrer et que d'autres se battent pour en conserver la mémoire! 
Photographie 3.

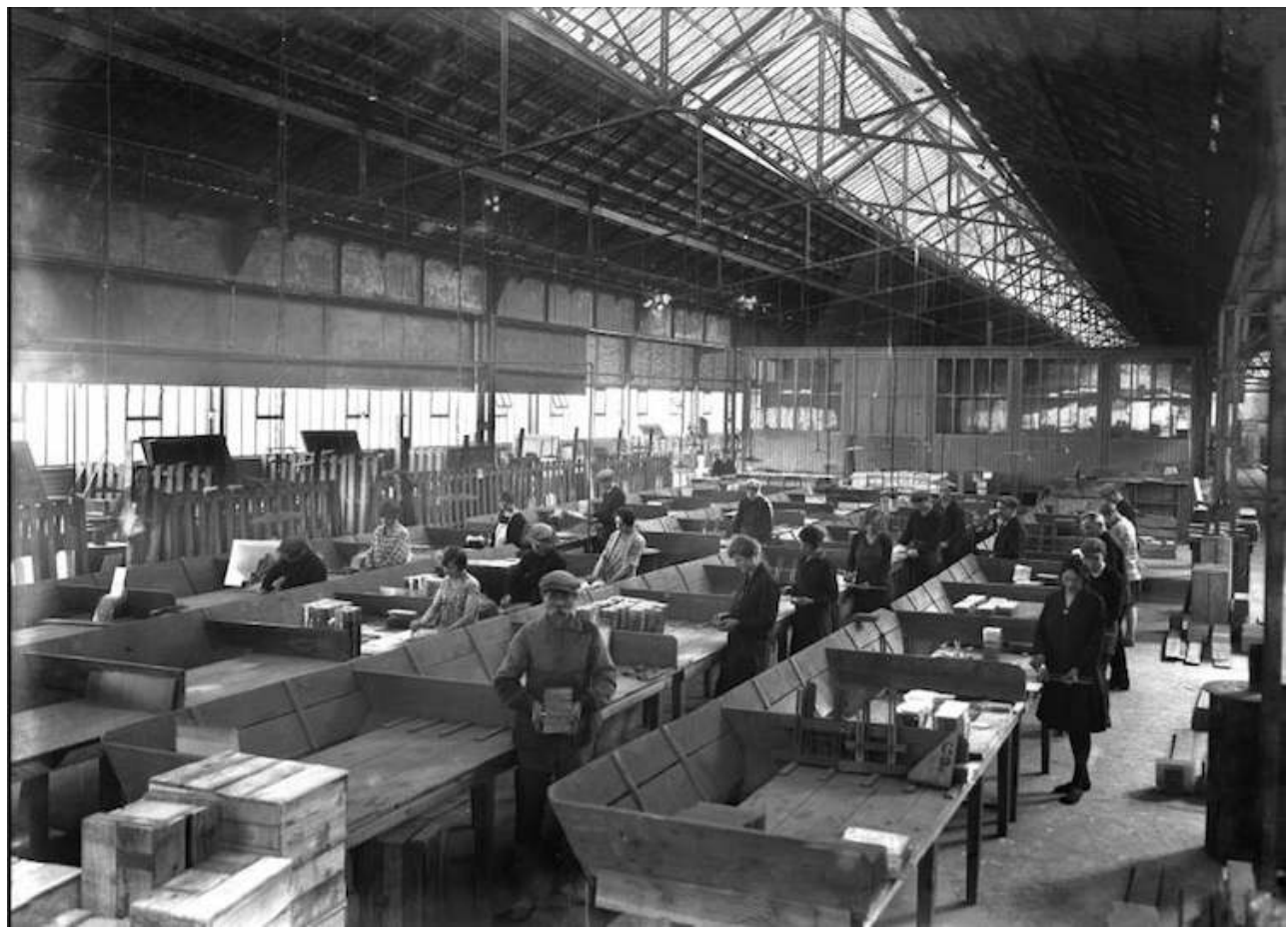

Aniche - Fabrication de plaques photographiques en verre chez Saint-Gobain à Aniche.

5 Nous ne proposerons pas ici de résumer les textes qui suivent mais nous invitons le lecteur à prendre le temps d'y plonger. Si Xavier Nerrière nous rappelle l'existence d'une archive ouvrière produite par les ouvriers eux-mêmes et suggère de s'y confronter pour en faire surgir de nouveaux questionnements, Jean Deilhes, prenant appui sur une série de photographies d'ouvriers réalisées par Dominique Delpoux, montre comment resurgit, derrière des portraits actuels, l'image encore vive dans les mémoires des mineurs d'autrefois tandis que les images montrent l'effet du travail sur les corps alors que les regards de ces ouvriers, montrés dans des lieux d'art, nous interrogent. Jan Middelbos s'interroge, précisément, sur les modes de représentation artistiques du monde du travail et nous met en garde contre une représentation qui oublierait les présupposés inhérents à la représentation et ferait l'impasse sur les potentialités transformatrices des formes d'auto-représentation. Géraldine Millo nous explique que " photographier la classe ouvrière aujourd'hui impose de comprendre les mutations dans lesquelles elle est prise pour ne pas reproduire à l'infini les poncifs construits sur une cristallisation de la grande époque.» Prenant appui sur les travaux d'Allan Sekula en particulier, de Philippe Bazin mais aussi sur des photographies produites par elle-même, l'auteur valorise les apprentissages réalisés par les jeunes lycéens qu'elle a photographiés et tend à rendre compte de ce qu'est devenue l'école des ouvriers. Sylvain Maresca, enfin, évoque cet «avenir en bleu » des jeunes de lycée professionnel, auquel ceux-ci se confrontent concrètement lorsqu'ils doivent endosser la tenue de travail typique de l'ouvrier tandis que leurs comportements révèlent l'ambivalence de leur rapport à l'héritage ouvrier. 


\section{BIBLIOGRAPHIE}

Azémar G-P. (1992), Ouvriers, ouvrières, un continent morcelé et silencieux, Autrement, $\mathrm{n}^{\circ} 126$.

Barthes R. (1980), La chambre claire, note sur la photographie, Paris, Seuil.

Benjamin W. (1935/2003), Petite histoire de la photographie, Paris, Alia (pour l'édition de 2002).

Rouillé A. (2005), La photographie, entre document et art contemporain, Paris, Gallimard, coll. Folio essais.

\section{AUTEUR}

\section{HENRI ECKERT}

Professeur de sociologie à l'Université de Poitiers et membre du GRESCO. 